\title{
COMPARISON RESULTS FOR PERIODIC BOUNDARY VALUE PROBLEM OF FRACTIONAL DIFFERENTIAL EQUATIONS
}

\author{
JUAN J. NIETO
}

Abstract. We study a linear fractional differential equation with a periodic boundary condition and give the explicit form of the solution and the corresponding Green's function. Using some properties of the Green's function we present some new comparison results.

Mathematics subject classification (2010): 34A08, 34B27, 34C25. result.

Keywords and phrases: Fractional differential equation, periodic boundary value problem, comparison

\section{REFERENCES}

[1] B. AhmAD, J.J. Nieto, Existence results for a coupled system of nonlinear fractional differential equations with three-point boundary conditions, Comput. Math. Appl., 58 (2009), 1838-1843.

[2] D. Araya, C. Lizama, Almost automorphic mild solutions to fractional differential equations, Nonlinear Anal., 69 (2008), 3692-3705.

[3] M. BELMEKKI, J.J. NieTo, R. RODRÍGUEZ-LÓPEZ, Existence of periodic solutions for a nonlinear fractional differential equation, Boundary Value Problems, 2009 (2009), Art. ID. 324561.

[4] B. Bonilla, M. Rivero, L. Rodríguez-Germá, J.J. Trujillo, Fractional differential equations as alternative models to nonlinear differential equations, Appl. Math. Comput., 187 (2007), 79-88.

[5] Y.-K. Chang, J.J. Nieto, Some new existence results for fractional differential inclusions with boundary conditions, Math. Comput. Modelling, 49 (2009), 605-609.

[6] K. Diethelm, N. J. Ford, Analysis of fractional differential equations, J. Math. Anal. Appl., 265 (2002), 229-248.

[7] A.A. Kilbas, H.M. SRivastava, J.J. Trujillo, Theory and Applications of Fractional Differential Equations, Elsevier Science B.V., Amsterdam, 2006.

[8] V. KiRYakova, Generalized Fractional Calculus and Applications, Longman Scientific \& Technical, Harlow; copublished in the United States with John Wiley \& Sons, Inc., New York, 1994.

[9] V. Laksmikantham, S. Leela, Nagumo-type uniqueness result for fractional differential equations, Nonlinear Anal., 71, 8 (2009), 2886-2889.

[10] V. Lakshmikantham, A.S. Vatsala, Basic theory of fractional differential equations, Nonlinear Anal., 69 (2008), 2677-2682.

[11] V. Lakshmikantham, S. Leela, J. Vasundhara DeVi, Theory of Fractional Dynamic Systems, Cambridge Scientific Pub., Cambridge, U.K., 2009.

[12] K. S. Miller, B. Ross, An Introduction to the Fractional Calculus and Differential Equations, John Wiley, New York, 1993.

[13] K.S. Miller, S.G. SAmko, Completely monotonic functions, Integr. Transf. Spec. Funct., 12 (2001), $389-402$.

[14] J. J. NIETo, Differential inequalities for functional perturbations first-order ordinary differential equations, Appl. Math. Letters, 15 (2002), 173-179.

[15] K.B. Oldham And J. Spanier, The Fractional Calculus, Academic Press, New York, London, 1974.

[16] I. Podlubny, Fractional Differential Equation, Academic Press, San Diego, 1999. 
[17] H. POllard, The complete monotonic character of the Mittag-Leffler function $E_{\alpha}(-x)$, Bull. Amer. Math. Soc., 54 (1948), 1115-1116.

[18] S. G. Samko, A. A. Kilbas, O. I. Marichev, Fractional Integrals and Derivatives. Theory and Applications, Gordon and Breach, Yverdon, 1993.

[19] W.R. SCHNEIDER, Completely monotone generalized Mittag-Leffler functions, Expo. Math., 14 (1996), 3-16.

[20] Z. SHUQIN, Monotone iterative method for initial value problem involving Riemann-Liouville fractional derivatives, Nonlinear Anal., 71 (2009), 2087-2093. 